\title{
Numerical Solution of SIR Model of Dengue Fever
}

\author{
M. Khalid \\ Department of Mathematical Sciences \\ Federal Urdu University of Arts, Sciences \& Techonology \\ University Road, Karachi-75300, Pakistan
}

\author{
Mariam Sultana \\ Department of Mathematical Sciences \\ Federal Urdu University of Arts, Sciences \& Technology \\ University Road, Karachi-75300, Pakistan
}

\author{
Fareeha Sami Khan \\ Department of Mathematical Sciences \\ Federal Urdu University of Arts, Sciences \& Technology \\ University Road, Karachi-75300, Pakistan
}

\begin{abstract}
Dengue is a complex disease because of the link it forms between humans, mosquitoes, and several virus serotypes, including efficient strategies for vector survival strategies. For this reason, the understanding of various factors that influence the recurrence of Dengue has been an inescapable fight for policy makers and scientists alike. In this paper, the susceptible-infected-recovered (SIR) model of dengue fever is presented and solved by incorporating a new technique called the Perturbation Iteration Algorithm (PIA). Through this method, the solution is in the form of a convergent series with easily computable components. The results show that the PIA and RK4 were in outstanding conformity.
\end{abstract}

\section{Keywords:}

Dengue Fever, Vector Population, Perturbation Iteration Method, Rate of Correlation

\section{INTRODUCTION}

As medical research advancement grew at the end years 20th century and vaccinations, antibiotics, and improvement of life conditions became top priorities, it was expected that the spread of infectious diseases were going to be overcome. That didn't happen because in developed countries the efforts were concentrated on cancer and other incurable conditions. Even with the dawn of the new century, infectious diseases are still causing immense suffering and, consequently, a high mortality rate in third world countries. Malaria, Yellow fever, AIDS, Ebola and other names have left a deep scar on the history of humanity forever.

Out of all these diseases, Dengue fever is particularly widespread in Southeast Asia and is progressively taking over the world by growing countries that have tropical climate. It is transmitted to human beings by a mosquito belonging to the Genus Aedes and exists in two forms: the Dengue Fever (DF) or classic dengue and Dengue Hemorrhagic Fever (DHF) which has the ability evolve to a painful and nearly fatal form termed the Dengue Shock Syndrome (DSS). The most problematic aspect of dengue is the fact that it can be caused by four distinct serotypes known as DEN1, DEN2, DEN3 and DEN4. When a person gets infected by one of the four, will never be infected by the same one again, which is a phenomena homologus immunity. However, once the human subject is attacked by either of the four, he looses immunity to the other three in about 12 weeks; this is known as heterologus immunity. This makes the patient more susceptible to cave into Dengue Hemorrhagic Fever DHF.

Classic dengue (DF) can be recognized by a sudden fever without any respiratory issue, but including intense headache, which explains its nickname;"the break bone fever". The patient stays affected for three days which can stretch out to a week, but the virus stay benign that time. The hemorrhagic form DHF causes sudden fever, nausea, leading to vomiting and loosing consciousness due to low blood pressure, which is an eventual result of dehydration. The symptoms continue to last for two or three days, and can lead to the death of the patient. Therefore, stopping the second infection has a chief importance because there is a possibility of its evolution toward a fatal form. Up until now, the strategies of mosquito control through the use of insecticides has proved futile. Moreover, environmental deterioration, poverty, climatic changes, unsanitary habitats, and unmonitored urbanization contribute greatly as complimentary factors to the propagation of infectious illnesses, especially the dengue fever.

In contrast to malaria which is prevalent in rural areas, caused by a parasitic mosquito, and chiefly infects at nighttime, the dengue fever is the result of an interaction between susceptible individuals and the mosquitoes of Genus Aedes which contain one of the four serotypes. The two species we know of that are transmit dengue are Aedes Aegypti and Aedes Albopictus. The former is Anthropophilic and thrives in heavily populated cities, biting primarily during the day; the latter, meanwhile, inhabits rural areas. Consequently, the danger of dengue is twofold:(i) Even in the absence of fatal forms, and because of its wide spreading and its multiple serotypes, the disease breeds significant economic and social costs (absenteeism, immobilization debilitation, medication).(ii) The potential risk of evolution towards the hemorrhagic form and the Dengue Shock Syndrome with high economic costs and which may lead to death. 
In order to have a more comprehensive knowledge about these diseases and for the preparation of strategies, mathematic modeling becomes an important tool. The creation of the model and the possibility of a simulation with parameter estimation allows tests for sensitivity and comparison of conjunctures, [1].

For dengue fever in particular, the mathematical models we have found in the literature propose compartmental dynamics with Susceptible, Exposed, Infective, and Removed (immunized). In particular, the SEIRS [2] and SIR models [3] with only one or two viruses acting simultaneously were considered [4].

\section{THE SIR MODEL FOR DENGUE FEVER}

The SIR mathematical model generates the spread of serotype 1 of the dengue virus between vector and the subject. The model is based on the Susceptible, Infected, and Removed SIR model of infectious disease epidemiology, which was adopted by [5, 6]. The SIR model discusses two kinds of populations, a human population $N_{h}$ and a vector population $N_{v}$. The $N_{h}$ is distinguished into three groups: potential victims of with the dengue virus (susceptible; $S_{h}$ ), people who are already infected with dengue (infected; $I_{h}$ ), and recovered former patients (removed; $R_{h}$ ). The vector population of mosquitoes $N_{v}$ is divided into two groups: mosquitoes that may potentially become infected with the virus (susceptible; $S_{v}$ ) and ones that have been infected with the dengue virus (infected; $\left.I_{v}\right)$.

It is assumed in this model that a certain number of people in the population have already been infected by the virus while others have not. It is also considered that the transmission of the virus grows in the population, while the number of mosquitoes remains constant. Both people and mosquitoes are put in one group at a time. The rate of change in the total host population which may easily be infected over the time due to host population birth rate is $\mu_{k} N_{k}$; people in category $S_{h}$ have the probability of being infected with dengue virus at a rate $\frac{b \beta_{h} I_{v}}{N_{h}}$, where $b \beta_{h}$ is sufficient rate of correlation of vector population to human population. $\beta_{h}$ represents the probability of infection from an infected individual to a mosquito that is susceptible to infection, while $b$ represents the average number of bites per infected mosquito. Deaths of the susceptible host are represented by $\mu_{h} S_{h}$.

The rate of change in the number of the infected host depends on the host infected population. A death among the infected host population is represented by $\mu_{h} I_{h}$, while members of the host population that recovers their health after infection is $\gamma_{h} I_{h}$. In addition, the total host population that has recovered $R_{h}$ will change according to changing times. The rate changes for a healthy population of the total time is the difference of the host recovers from infection $\gamma_{h} I_{h}$ with total mortality in healthy host $\mu_{h} R_{h}$.

Changes for the group $S_{v}$ show that each individual in the susceptible population has a probability of being bitten by mosquitoes infected with dengue virus at a rate $\frac{b \beta_{h} I_{v}}{N_{h}}$, where $b \beta_{v}$ is the sufficient rate of correlation of human to vector, including the probability of transmission from infected humans to potentially infected mosquitoes $\beta_{v}$. The number of deaths among the susceptible mosquito population is $\mu_{v} S_{v}$ at any given time, and total mortality of the population of infected mosquitoes is $\gamma_{v} I_{v}$. Changes that occur in all groups of people and of mosquitoes can be defined in a mathematical model of host-vector interaction comprising non-linear differential equations as follows:

$$
\begin{gathered}
\frac{d S_{h}}{d t}=\mu_{h} N_{h}-\frac{b \beta_{h} I_{v} S_{h}}{N_{h}}-\mu_{h} S_{h} \\
\frac{d I_{h}}{d t}=\frac{b \beta_{h} I_{v} S_{h}}{N_{h}}-\left(\mu_{h}+\gamma_{h}\right) I_{h} \\
\frac{d R_{h}}{d t}=\gamma_{h} I_{h}-\mu_{h} R_{h}
\end{gathered}
$$

Vector population

$$
\begin{gathered}
\frac{d S_{v}}{d t}=\mu_{v} N_{v}-\frac{b \beta_{v} I_{h} S_{v}}{N_{h}}-\mu_{v} S_{v} \\
\frac{d I_{v}}{d t}=\frac{b \beta_{v} I_{h} S_{v}}{N_{h}}-\mu_{v} I_{v}
\end{gathered}
$$

with initial condition,

$$
S_{h}+I_{h}+R_{h}=N_{h} \Rightarrow R_{h}=N_{h}-S_{h}-I_{h}
$$

and

$$
S_{v}+I_{v}=N_{v}=\frac{A}{\mu_{v}} \Rightarrow S_{v}=N_{v}-I_{v}=\frac{A}{\mu_{v}}-I_{v}
$$

Thus, the model for the human and mosquito populations can be simplified this way:

$$
\begin{gathered}
\frac{d S_{h}}{d t}=\mu_{h} N_{h}-\frac{b \beta_{h} I_{v} S_{h}}{N_{h}}-\mu_{h} S_{h} \\
\frac{d I_{h}}{d t}=\frac{b \beta_{h} I_{v} S_{h}}{N_{h}}-\left(\mu_{h}+\gamma_{h}\right) I_{h} \\
\frac{d I_{v}}{d t}=\frac{b \beta_{v} I_{h} S_{v}}{N_{h}}-\mu_{v} I_{v}
\end{gathered}
$$

The model can be simplified by assuming the following fractions: $x=\frac{S_{h}}{N_{h}}, y=\frac{I_{h}}{N_{h}}=\frac{I_{v}}{N_{v}}=\frac{I_{v}}{A / \mu_{v}}$ and $z=\frac{I_{v}}{N_{h}}$

\section{PERTURBATION ITERATION METHOD}

A novel and advanced iterative method called the "PerturbationIteration Method" has been derived recently by [7]. This new method masterfully uses a combination of perturbation expansions and taylor series expansions to derive an iteration scheme. Authors in [7. 8] presented expansion and correction terms of only first derivatives in the taylor Series expansion, i.e. $n=1, m=1$ and one correction term in the perturbation. The algorithm is named $P I A(1,1)$. Have a look at the following system of first-order differential equations.

$$
Y_{k}\left(\dot{x}_{k}, x_{j}, \epsilon, t\right)=0 ; k=1,2, . ., K ; j=1,2, . ., K
$$

where $k$ is a representative of the number of differential equations in the system and the number of dependent variables. $k=1$ for a single equation. In the open form, the system of equations is

$$
\begin{aligned}
Y_{1}= & Y_{1}\left(\dot{x}_{1}, x_{1}, x_{2}, x_{3}, \ldots, x_{K}, \epsilon, t\right)=0 \\
Y_{2}= & Y_{2}\left(\dot{x}_{2}, x_{1}, x_{2}, x_{3}, \ldots, x_{K}, \epsilon, t\right)=0 \\
Y_{3}= & Y_{3}\left(\dot{x}_{3}, x_{1}, x_{2}, x_{3}, \ldots, x_{K}, \epsilon, t\right)=0 \\
& \cdot \\
& \cdot \\
& \cdot \\
Y_{K}= & Y_{K}\left(\dot{x}_{K}, x_{1}, x_{2}, x_{3}, \ldots, x_{K}, \epsilon, t\right)=0
\end{aligned}
$$


Assume an approximate solution of the system

$$
x_{k, n+1}=x_{k, n}+\epsilon x_{k, n}^{c}
$$

with one correction term in the perturbation expansion. The subscript $n$ represents the $n^{\text {th }}$ iteration over this approximate solution. The system can be approximated with a Taylor series expansion in the neighborhood of $\epsilon=0$ as

$$
Y_{k}=\sum_{m=0}^{M} \frac{1}{m !}\left[\left(\frac{d}{d \epsilon}\right)^{m} Y_{k}\right]_{\epsilon=0} \times \epsilon^{m} ; k=1,2, . ., K
$$

where

$$
\frac{d}{d \epsilon}=\frac{\partial \dot{x}_{k, n+1}^{c}}{\partial \epsilon} \frac{\partial}{\partial \dot{x}_{k, n+1}}+\sum_{j=1}^{K}\left(\frac{\partial x_{j, n+1}^{c}}{\partial \epsilon} \frac{\partial}{\partial x_{j, n+1}}\right)+\frac{\partial}{\partial \epsilon}
$$

is defined for the $(n+1)^{t h}$ iterative equations

$$
Y_{k}\left(\dot{x}_{k, n+1}, x_{j, n+1}, \epsilon, t\right)=0
$$

substituting Eq. (8) into Eq.(7), obtain an iteration equation:

$$
\begin{aligned}
Y_{k}= & \sum_{m=0}^{M} \frac{1}{m !}\left[\left(\frac{\partial \dot{x}_{k, n+1}^{c}}{\partial \epsilon} \frac{\partial}{\partial \dot{x}_{k, n+1}}+\right.\right. \\
& \left.\left.\sum_{j=1}^{K}\left(\frac{\partial x_{j, n+1}^{c}}{\partial \epsilon} \frac{\partial}{\partial x_{j, n+1}}\right)+\frac{\partial}{\partial \epsilon}\right)^{m} Y_{k}\right]_{\epsilon=0} \times \epsilon^{m}=0
\end{aligned}
$$

where $k=1,2, . ., K$, which is a first-order differential equation and can be solved for the correction terms $x_{k, n}^{c}$. Then, using Eq. 10, the $(n+1)^{t h}$ iteration solution can be found. Iterations are terminated after a satisfactory approximation is obtained. For more detail and application of PIA, see [9.10].

\section{NUMERICAL SIMULATION}

In order to show the effectiveness of Perturbation Iteration Algorithm for solving the dynamical model of dengue fever, we present the following system of differential equation of Susceptible Infected Recovery (SIR) of dengue fever. The following is a system of differential equation of dengue fever

$$
\begin{gathered}
\frac{d x}{d t}=\mu_{h}(1-x(t))-\alpha x(t) z(t) \\
\frac{d y}{d t}=\alpha x(t) z(t)-\beta y(t) \\
\frac{d z}{d t}=\gamma(1-z(t)) y(t)-\delta_{1} z(t)
\end{gathered}
$$

where $\alpha=0.006, \beta=0.333333, \gamma=0.375, \delta_{1}=0.02941$, $\mu_{h}=0.0045, x\left(t_{\circ}\right)=c_{1}=\frac{5070822}{5071126}, y\left(t_{\circ}\right)=c_{2}=\frac{304}{5071126}$ and $z\left(t_{\circ}\right)=c_{3}=0.1$. The system is resolved using $P I A(1,1)$. Perturbation parameter is artificially introduced as

$$
\begin{gathered}
F_{1}=\dot{x}+\mu_{h} x(t)-\mu_{h}+\alpha x(t) z(t) \epsilon=0 \\
F_{2}=\dot{y}-\alpha x(t) z(t) \epsilon+\beta y(t)=0 \\
F_{3}=\dot{z}-\gamma y(t) \epsilon+\gamma y(t) z(t) \epsilon+\delta_{1} z(t)=0
\end{gathered}
$$

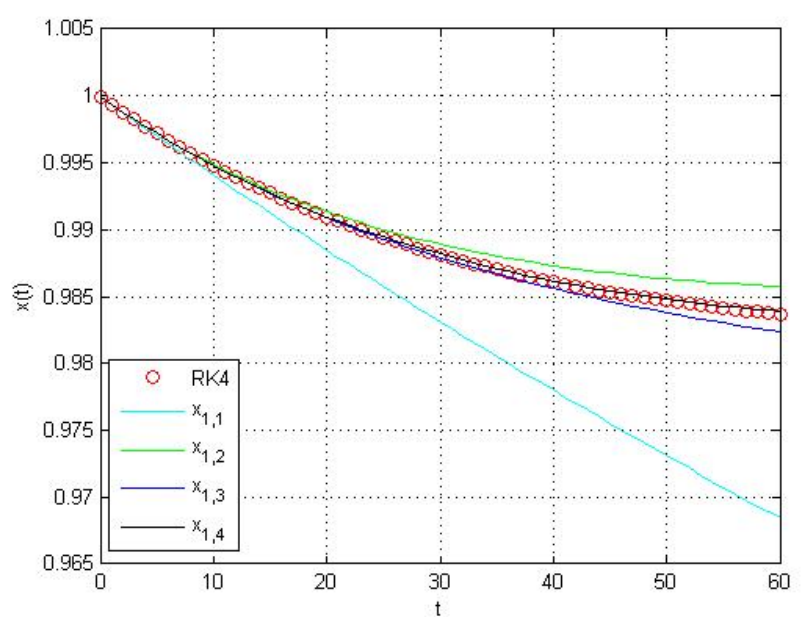

Fig. 1. Graphical representation of transmission of dengue virus in susceptible human population.

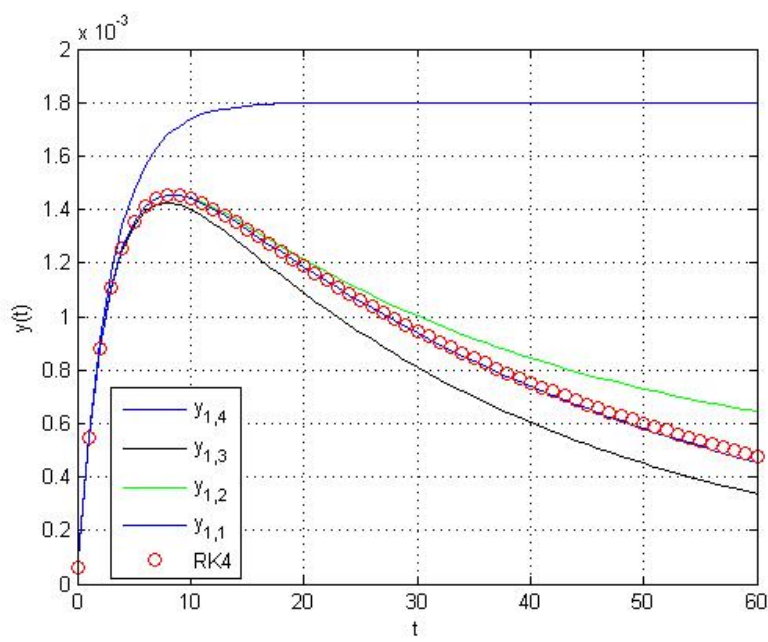

Fig. 2. Graph of transmission of dengue virus of infected human population.

for the Eq. 10 is reduced to

$$
\begin{gathered}
\dot{x}_{1, n}-\mu_{h}+\mu_{h} x_{1, n}+\dot{x}_{1, n}^{c} \epsilon+x_{1, n}^{c} \mu_{h} \epsilon+\alpha x_{1, n} z_{1, n} \epsilon=0 \\
\dot{y}_{1, n}+\beta y_{1, n}+\dot{y}_{1, n}^{c} \epsilon+\beta \dot{y}_{1, n}^{c}-\alpha x_{1, n} z_{1, n} \epsilon=0 \\
\dot{z}_{1, n}+\delta_{1} z_{1, n}+\dot{z}_{1, n}^{c} \epsilon+z_{1, n}^{c} \delta_{1} \epsilon+\gamma y_{1, n} z_{1, n} \epsilon-\gamma y_{1, n} \epsilon=0
\end{gathered}
$$

using initial trial functions are

$$
\begin{aligned}
x\left(t_{\circ}\right)= & 0.99994005276146 \\
y\left(t_{\circ}\right)= & 0.00005994723855 \\
& z\left(t_{\circ}\right)=0.1
\end{aligned}
$$




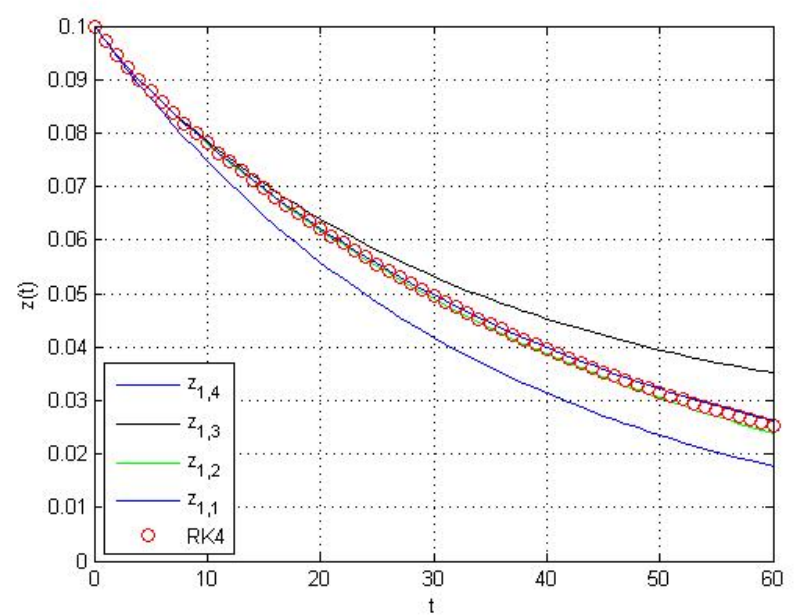

Fig. 3. Graphical representation of transmission of dengue fever in recovered human population.

$$
\begin{aligned}
x_{1,1}= & .9999365546+282.658 e^{-0.000046 t}- \\
& 282.658 \\
y_{1,1}= & 0.00006344538-0.039472 e^{-0.328886 t}+ \\
& 0.039472 e^{0.000001 t} \\
z_{1,1}= & 0.056+0.0553047 e^{-0.0323 t}-0.0553047
\end{aligned}
$$

The more iterations clearly shown in graph. Here, we don't write other iterations due to brevity.

\section{CONCLUSION}

In this research, the approximation of susceptible infected recovery model of dengue fever was investigated. For computations and plots, Mathematica 9.0 was employed. A comparison between Perturbation-Iteration Method and the fourth-order Runge-Kutta $R K 4$ method is mapped. This research work demonstrates that the Perturbation-Iteration Method has a great impact on the accuracy of efficient solution in this basic spread of dengue fever. Finally, we conclude that Perturbation-Iteration Method is a very reliable method for solving a broad array of dynamical problems due to its consistency used in a longer time frame and can be a helpful supplement in attaining a model for the dengue virus, in turn helping in eradicating the disease completely.

\section{Conflict of Interests}

The authors declare that there is no conflict of interests regarding the publication of this paper.

\section{Acknowledgment}

We thank the reviewers for their thorough efforts in editing our paper and highly appreciate the comments and constructive criticism that significantly contributed in improving the quality of the publication. The authors also thank Ms. Wishaal Khalid for proofreading our research paper.

\section{REFERENCES}

[1] Hethcote, H.W. (2000) The Mathematics of Infectious Diseases,SIAM, vol. 42, pp. 599-653.

[2] Newton, E.A. and Reiter, P. (1992) A model of the transmission of dengue fever with an evaluation of the impact of ultra-low volume (ULV) Insecticide applications on dengue epidemics. Am J Trop Med Hyg, vol. 47, pp. 709-720.

[3] Esteva, L. and Vargas, C. (1998) Analysis of a dengue disease transmission model, Mathematical Biosciences, vol. 150, pp. 131-151.

[4] Feng, Z. and Hernandez, V. (1997) Competitive exclusion in a vector-host model for the dengue fever, Journal of Mathematical Biology, vol. 35, pp. 523-544.

[5] Nuraini, N., Soewono, E. and Sidarto, K. (2007) Mathematical model of dengue disease transmission with severe dhf compartment, Bulletin of the Malaysian Mathematical Sciences Society, vol. 30(2), pp. 143-157.

[6] Yaacob, Y. (2007) Analysis of a dengue disease transmission model without immunity, MATEMATIKA Universiti Teknologi Malaysia, vol. 23(2), pp. 75-81.

[7] Aksoy, Y. and Pakdemirli, M. (2010) New perturbationiteration solutions for Bratu-type equations, Computers and Mathematics with Applications, vol. 59(8), pp. 28022808.

[8] Pakdemirli, M., Aksoy, Y. and Boyaci, H. (2011) A new perturbation-iteration approach for first order differential equations, Mathematical and Computational Applications, vol. 16(4), pp. 890-899.

[9] Khalid, M., Mariam, S. and Fareeha, S.K. (2015) A numerical computation of a model for HIV infection CD4+T-cell, International Journal of Innovation and Scientific Research, (In Press, Paper Key: IJISR-15-052-03).

[10] Khalid, M., Mariam, S., Faheem, Z. and Fareeha, S.K. (2015) Solving lakes system by using perturbation iteration method, International Journal of Computer Applications, vol. 114(4), pp. 1-7. 